\title{
Chapter 1 \\ The Use of Remote Sensing to Enhance Biodiversity Monitoring and Detection: A Critical Challenge for the Twenty-First Century
}

\author{
Jeannine Cavender-Bares, John A. Gamon, and Philip A. Townsend
}

\subsection{Introduction}

Improved detection and monitoring of biodiversity is critical at a time when Earth's biodiversity loss due to human activities is accelerating at an unprecedented rate. We face the largest loss of biodiversity in human history, a loss which has been called the "sixth mass extinction" (Leakey 1996; Kolbert 2014), given that its magnitude is in proportion to past extinction episodes in Earth history detectable from the fossil record. International efforts to conserve biodiversity (United Nations 2011) and to develop an assessment process to document changes in the status and trends of biodiversity globally through the Intergovernmental Science-Policy Platform on Biodiversity and Ecosystem Services (Díaz et al. 2015) have raised awareness about the critical need for continuous monitoring of biodiversity at multiple spatial scales across the globe. Biodiversity itself - the variation in life found among ecosystems and organisms at any level of biological organization-cannot practically be observed everywhere. However, if habitats, functional traits, trait diversity, and the spatial turnover of plant functions can be remotely sensed, the potential exists to globally inventory the diversity of habitats and traits associated with terrestrial biodiversity. To face this challenge, there have been recent calls for

\footnotetext{
J. Cavender-Bares $(\triangle)$

Department of Ecology, Evolution \& Behavior, University of Minnesota,

Saint Paul, MN, USA

e-mail: cavender@umn.edu

\section{J. A. Gamon}

Department of Earth \& Atmospheric Sciences, University of Alberta, Edmonton, AB, Canada

Department of Biological Sciences, University of Alberta, Edmonton, AB, Canada

CALMIT, School of Natural Resources, University of Nebraska - Lincoln, Lincoln, NE, USA

P. A. Townsend

Department of Forest and Wildlife Ecology, University of Wisconsin, Madison, WI, USA
} 
a global biodiversity monitoring system (Jetz et al. 2016; Proença et al. 2017; The National Academy of Sciences 2017). A central theme of this volume is that remote sensing (RS) will play a key role in such a system.

\subsection{Why a Focus on Plant Diversity?}

Plants and other photosynthetic organisms form the basis of almost all primary productivity on Earth, and their diversity and function underpin virtually all other life on this planet. Plants - collectively called vegetation-regulate the flow of critical biogeochemical cycles, including those for water, carbon, and nitrogen. They affect soil chemistry and other properties, which in turn affect the productivity and structure of ecosystems. Given the importance of plant diversity for providing the ecosystem services on which humans depend-including food production and the regulating services that maintain clean air and freshwater supply (Millennium Ecosystem Assessment 2005; IPBES 2018a) - it is critical that we monitor and understand plant biodiversity from local to global scales, encompassing genetic variation within and among species to the entire plant tree of life (Cavender-Bares et al. 2017; Jetz et al. 2016; Turner 2014).

Of the 340,000 known seed plants on Earth and the 60,000 known tree species (Beech et al. 2017), 1 out of every 5 seed plants and 1 out of every 6 tree species are threatened (Kew Royal Botanic Gardens 2016). Vulnerability to threats ranging from climate change to disease varies among species and lineages because of evolved differences in physiology and spatial proximity to threats. Across all continents, the largest threat to terrestrial biodiversity is land use change due to farming and forestry, while climate change, fragmentation, and disease loom as everincreasing threats (IPBES 2018a; b; c; d). Many plant species are at risk for extinction due to a combination of global change factors, including drought stress, exotic species, pathogens, land use change, altered disturbance regimes, application of chemicals, and overexploitation.

\subsection{The Promise of Remote Sensing to Detect Plant Diversity}

Different plants have evolved to synthesize different mixes of chemical compounds arranged in contrasting anatomical forms to support survival and growth. In addition, the structures of plant canopies correspond to different growth strategies in response to climate, environment, or disturbance. Differences among individual plants, populations, and lineages result from contrasting evolutionary histories, genetic backgrounds, and environmental conditions. Because these differences are readily expressed in aboveground physiology, biochemistry, and structure, many of these properties can be detected using spectral reflectance from leaves and plant canopies (Fig. 1.1). Plant pigments absorb strongly electromagnetic radiation in the 

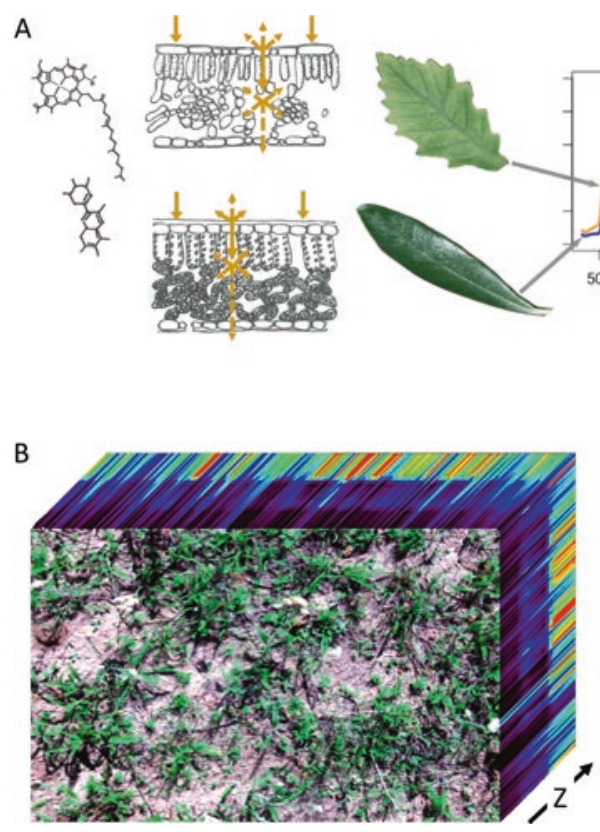

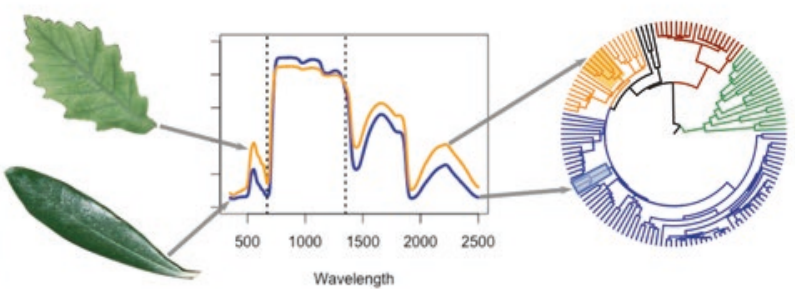

C

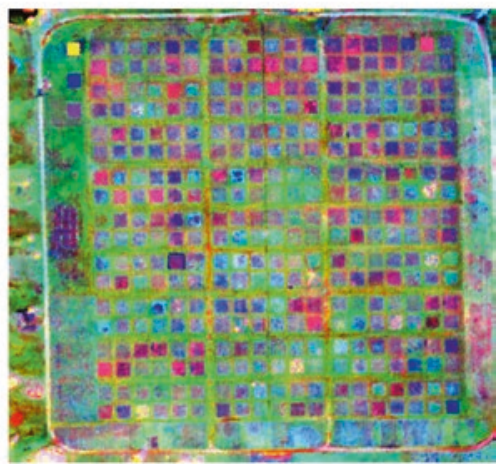

Fig. 1.1 (a) The chemical, structural, and anatomical attributes of plants influence the way they interact with electromagnetic energy to generate spectral reflectance profiles that reveal information about plant function and are tightly coupled to their evolutionary origins in the tree of life. (Adapted from Cavender-Bares et al. 2017.) Imaging spectroscopy offers the potential to remotely detect patterns in diversity and chemical composition and vegetation structure that inform our understanding of ecological processes and ecosystem functions. Examples are shown from the Cedar Creek Ecosystem Science Reserve long-term biodiversity experiment. (b) The image cube $(0.5 \mathrm{~m} \times 1 \mathrm{~m})$ at $1 \mathrm{~mm}$ spatial resolution $(400-1000 \mathrm{~nm})$ detects sparse vegetation early in the season in which individual plants can be identified. The "Z-dimension" (spectral dimension) illustrates different spectral reflectance properties for different scene elements, including different species. At this spatial resolution, plant diversity is predicted from remotely sensed spectral diversity (Wang et al. 2018). (c) AVIRIS NextGen false color image of the full experiment at $1 \mathrm{~m}$ spatial resolution (400-2500 nm). Each square is a $9 \times 9 \mathrm{~m}$ plot with a different plant composition and species richness. Wang et al. (2019) mapped chemical composition and a suite of other functional traits and their uncertainties in all of the experimental plots. By combining spectral data at different scales, proximal and remote imagery can be used to examine the scale dependence of the spectral diversity-biodiversity relationship in detail (e.g., Wang et al. 2018; Gamon et al. Chap. 16)

visible wavelengths (400-700 nm), while other chemical compounds and structural attributes of plants that tend to be conserved through evolutionary history affect longer wavelengths. The patterns of light absorbed, transmitted, and reflected at different wavelengths from vegetation reveal leaf and canopy surface properties, tissue chemistry, and anatomical structures and morphological attributes of leaves, whole plants, and canopies. Thus, technological advances for assessing optical properties of plants provide profound opportunities for detecting functional traits of organisms at different levels of biological organization. These advances are occurring at multiple spatial scales, with technologies ranging from field spectrometers and airborne 
systems to emerging satellite systems. As a consequence, there is high potential to detect and monitor plant diversity — and other forms of diversity_across a range of spatial scales, and to do so iteratively and continuously, particularly if multiple methods can be properly coordinated.

Calls for a global biodiversity observatory (Fig. 1.2) that can detect and monitor functional plant diversity from space (Jetz et al. 2016; Proença et al. 2017; The National Academy of Sciences 2017; Geller et al., Chap. 20) have been met with widespread support. Forthcoming satellite missions, including the Surface Biology and Geology (SBG) mission in planning stages at the US National Aeronautics and Space Administration (NASA) and related missions in Europe and Japan (Schimel et al., Chap. 19), will make unprecedented spectroscopic data available to scientists, management communities, and decision-makers, but at relatively coarse spatial scales. At the same time, rapid progress is being made with field spectroscopy using unmanned aerial vehicles (UAVs) and other airborne platforms that are offering novel ways to use RS to advance our understanding of the linkages between optical (e.g., spectral or structural) diversity and multiple dimensions of biodiversity (e.g., species, functional, and phylogenetic diversity) at finer spatial scales (Fig. 1.1). These advances present a timely and tremendously important opportunity to detect changes in the Earth's biodiversity over large regions of the planet. One can fairly ask whether user communities are ready to make use of the data. Effective interpretation and application of remotely sensed data to determine the status and trends of plant biodiversity and plant functions across the tree of life-with linkages to all other living organisms - requires integration across vastly different knowledge arenas. Critically, it requires integration with in-situ direct and indirect measures of species distributions, their evolutionary relationships, and their functions. Approaches for integration are the primary focus of this book.

A central requirement to advance monitoring of biodiversity at the global scale is to decipher the sources of variation that contribute to spectral variation, both from a biological perspective and from a physical perspective. Distinct fields of biology have developed a range of methodologies for understanding plant ecological and evolutionary processes that underlie these sources of variation. Similarly, radiative transfer models have been developed largely based on the physics of light interacting with vegetation canopy elements and the atmosphere. These models have yet to capture the full range of plant traits, often preferring to represent "average" vegetation conditions for a region instead of the variation present, so are not yet ready for the task. All of these methods have unique approaches to analyzing complex, multidimensional data sets, and neither the analytical approaches nor the data structures have been brought together in a systematic or comprehensive manner. A common language among disciplines (including biology, physiological ecology, landscape ecology, genetics, phylogenetics, geography, spectroscopy, and radiative transfer) related to the RS of biodiversity is currently lacking. This book provides a framework for how biodiversity, focusing particularly on plants, can be detected using proximally and remotely sensed hyperspectral data (with many contiguous spectral bands) and other tools, such as lidar (with its ability to detect structure). The chapters in this book present a range of perspectives and approaches on how RS can be 


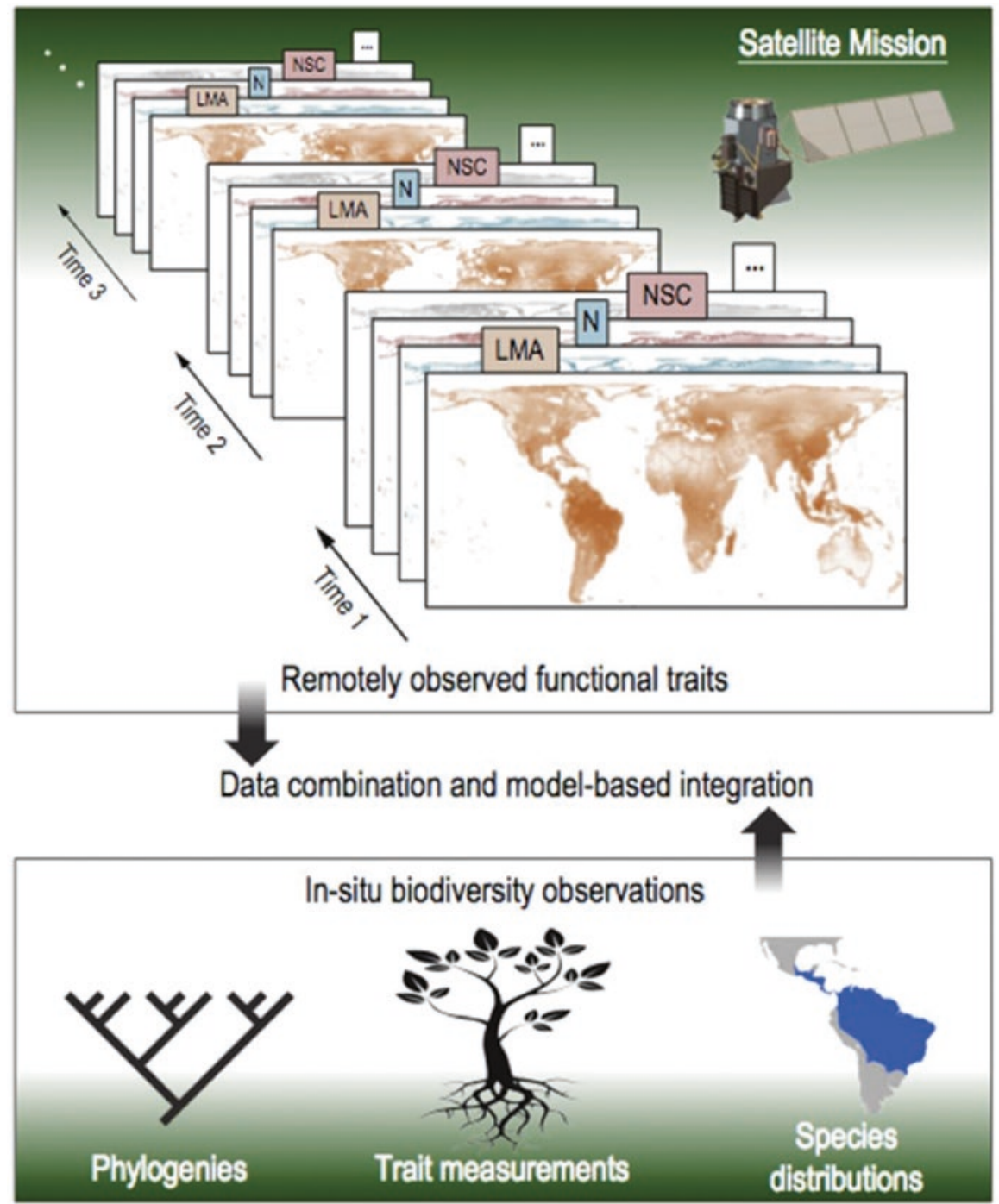

Fig. 1.2 An envisioned global biodiversity observatory in which remotely sensed high spectral resolution spectroscopic data from satellites is integrated with biodiversity observations through natural history studies, phylogenetic systematics, functional trait measurements, and species distribution data. The figure is adapted from Jetz et al. 2016 based on the National Center for Ecological Analysis and Synthesis Working Group "Prospects and priorities for satellite monitoring of global terrestrial biodiversity"

integrated to detect and monitor the status and trends of plant diversity, as well the biodiversity of other organisms and life processes that depend on plants above- and belowground. Biological and computational experts from three disciplines- RS and leaf optics, plant functional biology, and systematics-present insights to 
advance our understanding of how to link spectral and other kinds of RS data with functional traits, species distributions, and the tree of life for biodiversity detection. The authors detail the approaches and conditions under which efforts to detect plant biodiversity are likely to succeed, being explicit about the advantages and disadvantages of each. A theme running through many chapters is the challenge of moving across spatial scales from the leaf level to the canopy, ecosystem, and global scale. We provide a glossary that allows a common language across disciplines to emerge.

Here we explore the prospects for integrating components from each of these fields to remotely detect biodiversity and articulate the major challenges in our ability to directly link spectral data of vegetation to species diversity, functional traits, phylogenetic information, and functional biodiversity at the global scale. RS offers the potential to fill in data gaps in biodiversity knowledge locally and globally, particularly in remote and difficult-to-access locations, and can help define the larger spatial and temporal background needed for more focused and effective local or regional studies. It also may increase the likelihood of capturing temporal variation, and it allows monitoring of biodiversity at different spatial scales with different platforms and approaches. In essence, it provides the context within which changing global biodiversity patterns can be understood. The concept of "optical surrogacy" (Magurran 2013) - in which the linkage of spectral measurements to associated patterns and processes is used-may be useful in predicting ecosystem processes and characteristics that themselves are not directly observable (Gamon 2008; Madritch et al. 2014; Fig. 1.3). In a broad sense, such relationships between various expressions of biodiversity and optical (spectral) diversity provide a fundamental principle for "why RS works" as a metric of biodiversity and why so many different methods at different scales can provide useful information.

\subsection{The Contents of the Book}

The first section of the book presents the potential and basis for direct and indirect remote detection of biodiversity.

Cavender-Bares et al. (Chap. 2) present an overview of biodiversity itself, including the in-situ methods and metrics for measuring biodiversity, particularly plant diversity. The chapter provides a layperson's overview of the elements, methods, and metrics for detecting and analyzing biodiversity and points to the potential of spectral data, collected at multiple spatial and biological scales, to enhance the study of biodiversity. In doing so, it bridges an ecological and evolutionary understanding of the diversity of life, considering both its origins and consequences.

Serbin and Townsend (Chap. 3) describe various approaches for measuring plant and ecosystem function using spectroscopy, providing both the historical development of past advances and the potential of these approaches looking forward. The chapter explains why we are able to retrieve functional traits from spectra, which traits can be retrieved, and where spectra show features important for different aspects of plant function. It also raises the challenge of scaling plant function from leaves to canopies and landscapes. 

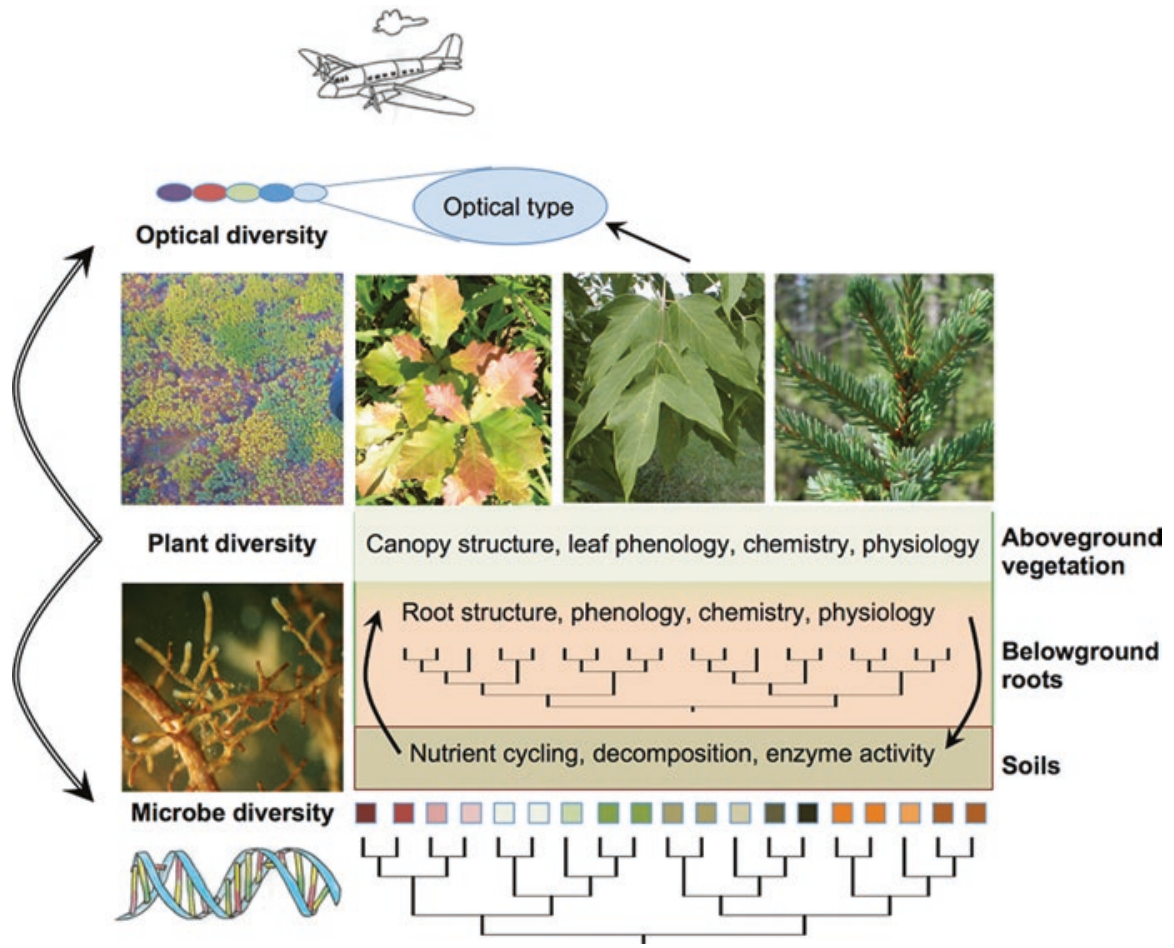

Fig. 1.3 Optical methods for detecting the functional, structural, and chemical components of vegetation, which are tightly coupled to the genetic and phylogenetic backgrounds of plants, are linked to belowground processes and the structure and function of microbial communities

Morsdorf et al. (Chap. 4) then present the Laegeren forest site in Switzerland as a virtual laboratory. They demonstrate how spectroscopy can be operationalized for RS of functional diversity to explain plant biodiversity patterns and ecosystem functions. The Laegeren site is one of the best-studied sites in the world for this purpose and is used as a case study to explain ground truthing and what can be learned from landscape-level detection of functional diversity.

Martin (Chap. 5) summarize the experiences with "spectronomics"-a framework aimed at integrating chemical, phylogenetic, and spectral RS data-using airborne imagery to detect forest composition and function in wet tropical forests. In these vast, largely inaccessible landscapes that harbor enormous taxonomic variation, approaches that rely solely on field-based observations are infeasible, illustrating an essential role for RS. As pioneers in using spectroscopy to detect plant chemistry, function, and biodiversity in tropical forests around the world, these researchers highlight some of the major lessons they have learned.

Pontius et al. (Chap. 6) consider how biodiversity can be protected given current threats to forest and vegetation conditions and present approaches for detailed and accurate detection of forest disturbance and decline. They review current techniques used to assess and monitor forest ecosystem condition and disturbance and outline 
a general approach for earlier, more detailed and accurate decline assessment. They also discuss the importance of engaging land managers, practitioners, and decisionmakers in these efforts to ensure that the products developed can be utilized by stakeholders to maximize their impact.

Meireles et al. (Chap. 7) provide a framework to explain how spectral reflectance data from plants is tightly coupled to the tree of life and demonstrate how spectra can reveal evolutionary processes in plants. They clarify that many spectral features in plants are inherited and are thus very similar among close relatives-in other words, they are highly phylogenetically conserved. Simulations reveal that spectral information of plants appears to follow widely used evolutionary models, making it possible to link plant spectra to the tree of life in a predictive manner. As a consequence, methods developed in evolutionary biology to understand the tree of life can now benefit the RS community. The chapter provides evidence that evolutionary lineages may be easier than individual species to detect through RS methods, particularly if they are combined with other approaches for estimating which species and lineages have the potential to be present in a given location. A caveat is that spatial resolution of satellite spectral data will limit such inferences, but leaf- and canopy-level spectra (obtainable from proximal and airborne sensing) can contribute enormously to our understanding of these fundamental links between spectral patterns and gene sequences.

Madritch et al. (Chap. 8) link aboveground plant biodiversity and productivity to belowground processes. They explain the functional mechanisms-which can be revealed by remotely sensed spectral data-that influence interactions of plant hosts with insects and soil organisms, in turn influencing ecosystem functions, such as decomposition and nutrient cycling. The chapter provides an example of using the concept of surrogacy, in which the biochemical linkage of spectral measurements to associated patterns and processes aboveground is used to provide estimates of soil and microbial processes belowground that are not directly observable via RS.

The next three chapters focus on linking satellite-based remotely sensed data to biodiversity prediction. Pinto-Ledezma and Cavender-Bares (Chap. 9) present an example of how currently available satellite-based RS products can be used to generate next-generation species distribution models to predict where species and lineages are likely occur and the habitats they may have access to and persist in under altered climates in the future. They compare RS-based methods for generating predictive models with widely used approaches that use meteorologically derived climate variables. They demonstrate the advantages of RS-based models in regions where meteorological data is only sparsely available. Such predictive modeling that harnesses species occurrence data and temporal information about the biotic environment may make spectral methods of species and evolutionary lineage detection more tractable.

Building on the availability of satellite RS data with near-global coverage to predict biodiversity, Record et al. (Chap. 10) explore how RS illuminates the relationship between biodiversity and geodiversity - the variety of abiotic features and processes that provide the template for the development of biodiversity. They introduce a variety of globally available geodiversity measures and examine how they can be combined with biodiversity data to understand how biodiversity responds to geodiversity. The authors use the analogy of the "stage" that defines the patterns of life to some 
degree, often measured as habitat heterogeneity, a key driver of species diversity. They illustrate the approach by examining the relationship between biodiversity and geodiversity with tree biodiversity data from the US Forest Inventory and Analysis Program and geodiversity data from remotely sensed elevation from the Shuttle Radar Topography Mission (SRTM). In doing so, they outline the challenges and opportunities for using RS to link biodiversity to geodiversity.

Paz et al. (Chap. 11) present an approach for using RS data to predict patterns of plant diversity and endemism in the tropics within the Brazilian Atlantic rainforest. They examine how RS environmental data from tropical regions can be used to support biodiversity prediction at multiple spatial, temporal, and taxonomic scales.

Bolch et al. (Chap. 12) summarize the range of approaches that can be used to optimize detection of invasive alien species (IAS), which pose severe threats to biodiversity. These approaches emphasize the ability to detect individual plant species that have distinct functional properties. The chapter presents current RS capabilities to detect and track invasive plant species across terrestrial, riparian, aquatic, and human-modified ecosystems. Each of these systems has a unique set of issues and species assemblages with its own detection requirements. The authors examine how RS data collection in the spectral, spatial, and temporal domains can be optimized for a particular invasive species based on the ecosystem type and image analysis approach. RS approaches are enhancing studies of the invasion processes and enabling managers to monitor invasions and predict the spread of IAS.

The next three chapters of the book explore how components of diversity can be detected spectrally and remotely with a focus on optical detection methods and technical challenges. Lausch et al. (Chap. 13) delve into the complexity of monitoring vegetation diversity and explain how no single monitoring approach is sufficient on its own. The chapter introduces the range of Earth observation (EO) techniques available for assessing vegetation diversity, covering close-range EO platforms, spectral approaches, plant phenomics facilities, ecotrons, wireless sensor networks (WSNs), towers, air- and spaceborne EO platforms, UAVs, and approaches that integrate air- and spaceborne EO data. The chapter presents the challenges with these approaches and concludes with recommendations and future directions for monitoring vegetation diversity using RS.

Ustin and Jacquemoud (Chap. 14) provide the physical basis for detecting the optical properties of leaves based on how they modify the absorption and scattering of energy to reveal variation in function. The chapter provides considerable detail on how the combination of absorption and scattering properties of leaves together creates the shape of their reflectance spectrum. It also reviews and summarizes the most common interactions between leaf properties and light and the physical processes that regulate the outcomes of these interactions.

Schweiger (Chap. 15) describes a set of best practices for planning field campaigns and collecting and processing data, focusing on spectral data of terrestrial plants collected across various levels of measurements, from leaf to canopy to airborne. These approaches also generally apply to RS of aquatic systems, soil, and the atmosphere and to active RS systems, such as lidar, thermal, and satellite data collection. Schweiger discusses how goals for data collection can be broadly classified into model calibration, model validation, and model interpretation. 
The final chapters of the book move to the issues of temporal and spectral scale and integration across scales. Gamon et al. (Chap. 16) present a thorough examination of the challenges in spectral methods for detecting biodiversity posed by issues of spatial, temporal, and spectral dimensions of scale. They explain why the size of the organism relative to the pixel size of detection has consequences for spectral detection of different components of biodiversity and draw on a rich history of literature on scaling effects, including geostatistical approaches for sampling across spatial scales. The chapter emphasizes the importance of developing biodiversity monitoring systems that are "scale-aware" as well as the value of an integrated, multi-scale sampling approach.

Schrodt et al. (Chap. 17) outline how environmental and socioeconomic data can be integrated with biodiversity and RS data to expand knowledge of ecosystem functioning and inform biodiversity conservation decisions. They present the concepts, data, and methods necessary to assess plant species and ecosystem properties across spatial and temporal scales and provide a critical discussion of the major challenges.

Fernández et al. (Chap. 18) provide a framework for understanding Essential Biodiversity Variables (EBVs) to integrate in-situ biodiversity observations and RS through modeling. They argue that open and reproducible workflows for data integration are critical to ensure traceability and reproducibility to allow each EBV to be updated as new data and observation systems become available. The chapter makes the case that the development of a globally coordinated system for biodiversity monitoring will require the mobilization of and integration of in-situ biodiversity data not yet publicly available with emerging RS technologies, novel biodiversity models, and informatics infrastructures.

Schimel et al. (Chap. 19) discuss the prospects and pitfalls for RS of biodiversity at the global scale, focusing on imaging spectroscopy and NASA's Surface Biology and Geology mission concept.

Finally, Geller et al. (Chap. 20) provide an epilogue to the book and present a vision for a global biodiversity monitoring system that is flexible and accessible to a range of user communities. Such a system will require a coordinated effort among space agencies, the RS community, and biologists to bring information about the status and trends in biodiversity, ecosystem functions, and ecosystem services together so that different data streams inform each other and can be integrated. The chapter explains that the Group on Earth Observations Biodiversity Observation Network (GEO BON), the International Long Term Ecological Research Site (ILTER) network, the US National Ecological Observatory Network (NEON), and a variety of sponsors and other organizations are working to enhance coordination and to develop guidelines and standards that will serve this vision.

Indeed, a rapidly advancing global movement has emerged with a shared vision to develop the capacity to monitor the status and trends in the Earth's biodiversity. The authors of this book have sought to contribute to that shared vision through their varied perspectives and experiences. Collectively, the chapters present a range of approaches and knowledge that can transform the ability of humanity to detect and interpret the changing functional biodiversity of planet Earth. 


\subsection{The Origins of the Book}

Before closing, we offer a note of acknowledgment on how this book came into existence. The editors, who themselves have contrasting backgrounds spanning several disciplines, were collaboratively funded, starting in 2013, by the US National Science Foundation (NSF) and the NASA Dimensions of Biodiversity program on the project Linking remotely sensed optical diversity to genetic, phylogenetic and functional diversity to predict ecosystem processes (DEB-1342872,1342778). We worked together in several field sites for 5 years to advance our own understanding approaches for remote detection of plant biodiversity. The importance and high potential for rapid advances, as well as the need for the involvement of numerous experts, were obvious from the start. With support from the National Institute for Mathematical and Biological Synthesis (NIMBioS) for the working group on Remote Sensing of Biodiversity, we brought together many of the experts represented within the book, including a symposium in the fall of 2018 where authors shared their work and provided feedback to each other. Further interactions were fostered by the National Center for Ecological Analysis and Synthesis (NCEAS), annual meetings of the NASA Biological Diversity and Ecological Forecasting Program, the Keck Institute for Space Studies, the NSF Research Coordination Network on Biodiversity across scales, and bioDISCOVERY, an international research program fostering collaborative interdisciplinary activities on biodiversity and ecosystem science. bioDISCOVERY, which is part of Future Earth and is hosted and supported by the University Zürich's University Research Priority Program on Global Change and Biodiversity, provided generous financial support for the editing process. Additional financial support was provided by the NSF RCN (DEB: 1745562) and the Keck Institute for Space Studies. Mary Hoff served as technical editor for all of the chapters in the book. We express gratitude to all of these institutions and our many colleagues who contributed to the conception of this work along the way.

\section{References}

Beech E, Rivers M, Oldfield S, Smith PP (2017) GlobalTreeSearch: the first complete global database of tree species and country distributions. J Sustain For 36:454-489

Cavender-Bares J, Gamon JA, Hobbie SE, Madritch MD, Meireles JE, Schweiger AK, Townsend PA (2017) Harnessing plant spectra to integrate the biodiversity sciences across biological and spatial scales. Am J Bot 104:1-4. https://doi.org/10.3732/ajb.1700061

Díaz S, Demissew S, Carabias J, Joly C, Lonsdale M, Ash N, Larigauderie A, Adhikari JR, Arico S, Báldi A, Bartuska A, Baste IA, Bilgin A, Brondizio E, Chan KMA, Figueroa VE, Duraiappah A, Fischer M, Hill R, Koetz T, Leadley P, Lyver P, Mace GM, Martin-Lopez B, Okumura M, Pacheco D, Pascual U, Pérez ES, Reyers B (2015) The IPBES conceptual framework-connecting nature and people. Curr Opin Environ Sustain 14:1-16

Gamon JA (2008) Tropical remote sensing - opportunities and challenges. In: Kalacska M, Sanchez-Azofeifa GA (eds) Hyperspectral remote sensing of tropical and subtropical forests. CRC Press. Taylor and Francis Group, Boca Raton, pp 297-304 
IPBES (2018a) Summary for policymakers of the regional and subregional assessment of biodiversity and ecosystem services for Europe and Central Asia of the Intergovernmental SciencePolicy Platform on Biodiversity and Ecosystem Services. IPBES Secretariat, Bonn

IPBES (2018b) Summary for policymakers of the regional assessment report on biodiversity and ecosystem services for Africa of the Intergovernmental Science-Policy Platform on Biodiversity and Ecosystem Services. IPBES Secretariat, Bonn

IPBES (2018c) Summary for policymakers of the regional assessment report on biodiversity and ecosystem services for Asia and the Pacific of the Intergovernmental Science-Policy Platform on Biodiversity and Ecosystem Services. IPBES Secretariat, Bonn

IPBES (2018d) Summary for policymakers of the regional assessment report on biodiversity and ecosystem services for the Americas of the Intergovernmental Science-Policy Platform on Biodiversity and Ecosystem Services. IPBES Secretariat, Bonn

Jetz W, Cavender-Bares J, Pavlick R, Schimel D, Davis FW, Asner GP, Guralnick R, Kattge J, Latimer AM, Moorcroft P, Schaepman ME, Schildhauer MP, Schneider FD, Schrodt F, Stahl U, Ustin SL (2016) Monitoring plant functional diversity from space. Nature Plants 2:16024

Kew Royal Botanic Gardens (2016) State of the World's plants. Board of Trustees of the Royal Botanic Gardens, Kew, London, UK

Kolbert E (2014) The sixth extinction: an unnatural history. Henry Holt \& Company, New York

Leakey R, Roger L (1996) The sixth extinction: patterns of life and the future of humankind. Weidenfeld and Nicolson, London

Madritch MD, Kingdon CC, Singh A, Mock KE, Lindroth RL, Townsend PA (2014) Imaging spectroscopy links aspen genotype with below-ground processes at landscape scales. Philos Trans R Soc Lond B Biol Sci 369:20130194

Magurran AE (2013) Measuring biological diversity. Wiley-Blackwell, Malden

Millennium Ecosystem Assessment (2005) Ecosystems and human well-being: current state \& trends assessment. Island Press, Washington, D.C.

Proença V, Martin LJ, Pereira HM, Fernandez M, McRae L, Belnap J, Böhm M, Brummitt N, García-Moreno J, Gregory RD, Honrado JP, Jürgens N, Opige M, Schmeller DS, Tiago P, van Swaay CAM (2017) Global biodiversity monitoring: from data sources to essential biodiversity variables. Biol Conserv 213:256-263

Turner W (2014) Sensing biodiversity. Science 346:301

The National Academy of Sciences (2017) Thriving on our changing planet: a decadal strategy for earth observation from space. The National Academies Press, Washington, D.C.

United Nations (2011) Resolution 65/161. Convention on biological diversity

Wang R, Gamon JA, Cavender-Bares J, Townsend PA, Zygielbaum AI (2018) The spatial sensitivity of the spectral diversity-biodiversity relationship: an experimental test in a prairie grassland. Ecol Appl 28:541-556

Wang Z, Townsend PA, Schweiger AK, Couture JJ, Singh A, Hobbie SE, Cavender-Bares J (2019) Mapping foliar functional traits and their uncertainties across three years in a grassland experiment. Remote Sensing of Environment 221:405-416.

Open Access This chapter is licensed under the terms of the Creative Commons Attribution 4.0 International License (http://creativecommons.org/licenses/by/4.0/), which permits use, sharing, adaptation, distribution and reproduction in any medium or format, as long as you give appropriate credit to the original author(s) and the source, provide a link to the Creative Commons license and indicate if changes were made.

The images or other third party material in this chapter are included in the chapter's Creative Commons license, unless indicated otherwise in a credit line to the material. If material is not included in the chapter's Creative Commons license and your intended use is not permitted by statutory regulation or exceeds the permitted use, you will need to obtain permission directly from the copyright holder.

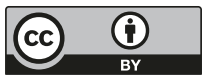

